\title{
A parallel-elastic actuation approach for wide bandwidth fingertip haptic devices
}

\author{
Daniele Leonardis $(10) \cdot$ Giancarlo Santamato $•$ \\ Massimiliano Gabardi • Massimiliano Solazzi • \\ Antonio Frisoli
}

Received: 15 July 2021 / Accepted: 9 January 2022 / Published online: 6 February 2022

(C) The Author(s) 2022

\begin{abstract}
Wearable haptic devices are used to render sense of touch in different virtual reality, simulated or teleoperated environments. Design of these devices has to comply with dimensional constraints imposed by ergonomics and usability, introducing compromises between size of the actuators and features of the rendered haptic stimuli, such as peak forces and bandwidth. We propose a new design approach for wearable haptic devices resembling the micro-macro actuation concept: it is based on two distinct actuators with different mechanical reduction, coupled by an elastic element. We show this better matches the output range of the actuators to features of the signals used in typical haptic rendering. We investigated the approach through an analytical model, a numerical model, and physical experiments conducted after design and development of a working prototype. The theoretical and simulated models allow to better understand dynamic interaction of the system parts, and to define design guidelines for the development of the real device. The adopted design solutions were implemented and evaluated in the prototype of a highly wearable fingertip device. Final experimental results show how the implementation of the proposed method is capable of an effective haptic
\end{abstract}

D. Leonardis $(\bowtie) \cdot$ G. Santamato $\cdot$ M. Gabardi

M. Solazzi · A. Frisoli

Institute of Mechanical Intelligence, Scuola Superiore

Sant'Anna of Pisa, Pisa, Italy

e-mail: d.leonardis@ santannapisa.it

URL: https://www.santannapisa.it rendering, that better matches the desired frequency response.

Keywords Cutaneous feedback · Haptic .

Bandwidth · Fingertip · Wearable · Parallel elastic .

Touch

\section{Introduction}

Advances in Virtual Reality (VR) in terms of computer graphics, Head Mounted Displays (HMD), and tracking systems provide immersion of the user in rich and informative virtual environments. These systems have become compact, wearable and immediate to use, and can be exploited in a variety of applications such as gaming, simulation and training, educational. Yet, sense of touch is still largely excluded, although different scenarios, including robotic teleoperation, involve manipulation tasks and would greatly take advantage of it (i.e. training of surgeons, robotic tele-manipulation). For the artificial rendering of the sense of touch, the challenge is to replicate the rich and complex tactile stimulations by means of a compact device, or to find the most convenient trade-off between rendering capabilities and relevance of specific haptic cues in the manipulation tasks. Different haptic devices have been developed in the past decades, ranging from desktop devices to exoskeleton architectures. Regarding compact and wearable devices, recent literature is particularly rich. 
Fig. 1 The presented Parallel Elastic approach for a fingertip haptic device: a transmission scheme, $\mathbf{b}$ developed prototype

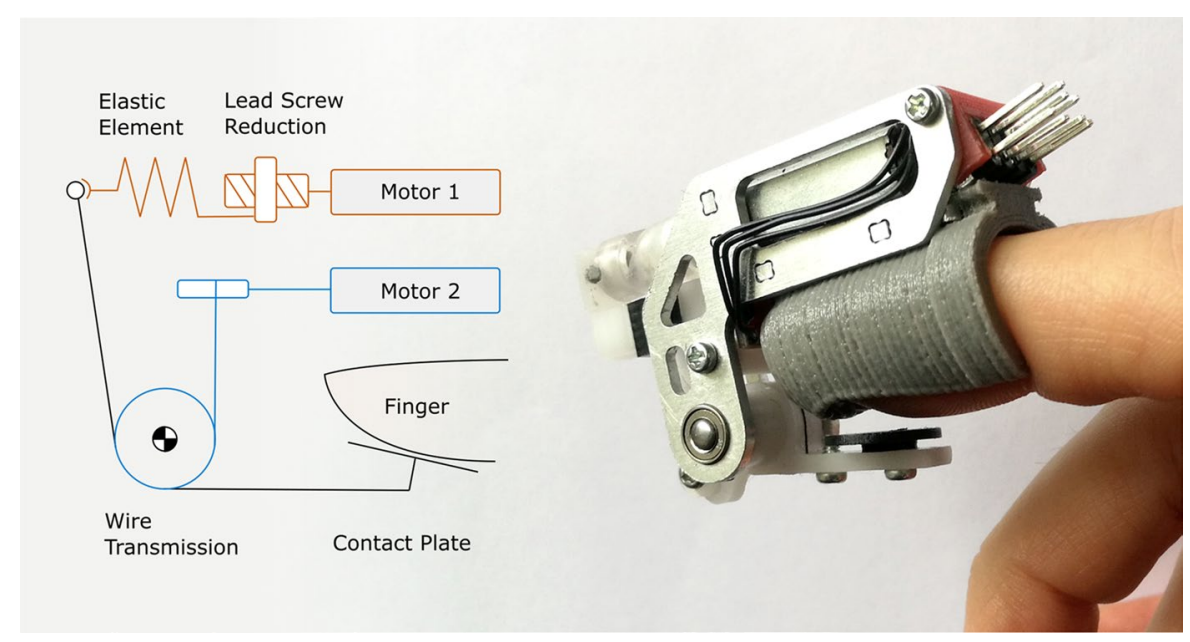

It proposes development of different devices capable of specific cutaneous feedback and cues, such as contact-no contact condition, modulation of grasping forces [19], contact orientation [3], area of contact [5], stretch and indentation [12], thermal [8], multimodal feedback $[6,21]$. A comprehensive review of wearable haptic devices including a taxonomy can be found in [17].

Rendering the variety of the tactile physical interaction and haptic cues is quite complex [2], and regarding wearable devices, practical constraints such as wearability, size and mass determine limits to the kind and range of stimuli that can be rendered. The actuator is usually the heaviest part of a wearable fingertip haptic interface. Typical DC motors or voice coils implemented in haptic devices allow for high quality haptic rendering, ranging from constant to high frequency force components, with the drawback of a limited maximum output force or a relatively heavy device [7]. When the above actuators are coupled with micro-sized gear reduction, they can amplify the output force in a very limited mass [13], yet at the cost of a severely reduced output bandwidth, and noise (due to gears) introduced in the rendered signal.

A solution proposed in literature [15] to increase the dynamic range of a robotic device is the micromacro approach, where a reduced motor (macro) is coupled to the output in parallel with a direct-drive motor (micro). The solution has been explored for grounded haptic devices in the shape of robotic manipulators arranged for haptic rendering [18] and [24]. In other haptic devices presented in literature, it is implemented in a series configuration where the "micro" actuator is placed at the end-effector of a desktop or grounded haptic device $[14,16,20]$ to enhance its output bandwidth. To the knowledge of authors, the parallel micro-macro approach has not been investigated yet to enhance performance of a wearable fingertip haptic device. In this paper, the proposed novel fingertip device implements a parallel elastic actuation system, obtained by coupling the output of two DC micro-motors of the same size: one motor with low reduction to increase the dynamic range of the device, the second with high mechanical reduction to increase the maximum static force (Fig. 1). This manuscript is an extended work of the preliminary conference paper [11].

\section{The parallel elastic approach}

Rationale of the proposed approach is originated by evaluation of the typical features of haptic rendering signals. In particular during tactile exploration and manipulation tasks, sources of the haptic interaction forces, and their magnitude and bandwidth, are different: one source component can be identified in voluntary movements of the user, including i.e. modulation of the grasping forces, or modulation of the pressing force when exploring a surface by touch. This source of interaction forces is characterized by a high maximum amplitude, and by low dynamics going from static (i.e. during stable grasping) to relatively low frequencies. In example, in [9] it shows grasping 
force adaptations in response to disturbances occur with time constants of about $0.2 \mathrm{~S}$.

Another source of interaction forces can be found in transients and vibrations caused by voluntary movements of the user: these are generated by contact transitions, friction and stick-slip conditions, texture, gratings, vibrations due to deformation and structural yelding of materials. These signals are usually characterized by lower amplitude and higher frequency range. Although with lower amplitude, fingerpads mechanoreceptors are highly sensitive to fast dynamic signals [1], up to the point the tactile sensory channel mixes with the auditory channel [23]. Perception of such high frequency haptic signals becomes very informative during virtual exploration [22] and telemanipulation [10].

On the basis of the above considerations, we investigated whether a micro-macro actuating method can better fit requirements of a fingertip haptic device. We further developed the method presented in [15] (designed there for an antropomorphic robotic arm) investigating its behavior for two identical actuators with different mechanical reduction and elastic coupling. Here we evaluate through a theoretical model, simulation results and experimental validation whether it was possible to better match actuators' output to the desired amplitude and dynamic response of the haptic rendering signals.

\subsection{Actuation architecture and design parameters}

The proposed parallel elastic actuation is represented in the scheme of Fig. 2a. The fingertip is modeled by a stiffness $k_{e}$ and a damping $c_{e}$. The fingertip is guided by a two branches link at a distance $l$ from the revolute joint $\Omega . \vartheta$ stands for the degree-of-freedom (dof) of the link, while $J_{p}$ stands for the moment of inertia about $\Omega$.

For the actuation, two identical DC micro motors have been considered. Motors dynamics includes the circuit resistance $R$, the torque constant $k_{T}$, the back electromotive force (BEMF) constant $k_{\phi}$, and the moment of inertia $J$. The counter electromotive effect is treated by attaching an equivalent damper $c=k_{T} k_{\phi} / R$ to the motor inertia.

One motor is equipped with a mechanical transmission that outputs a linear coordinate $x$, with a reduction ratio $\tau_{1}$. The connection between this first motor and the pinned link is implemented via an elastic element modeled by the lumped stiffness $k_{s}$ and and damping $c_{s}$. The lever arm of the elastic element is named $h$. Conversely, the second motor is coupled
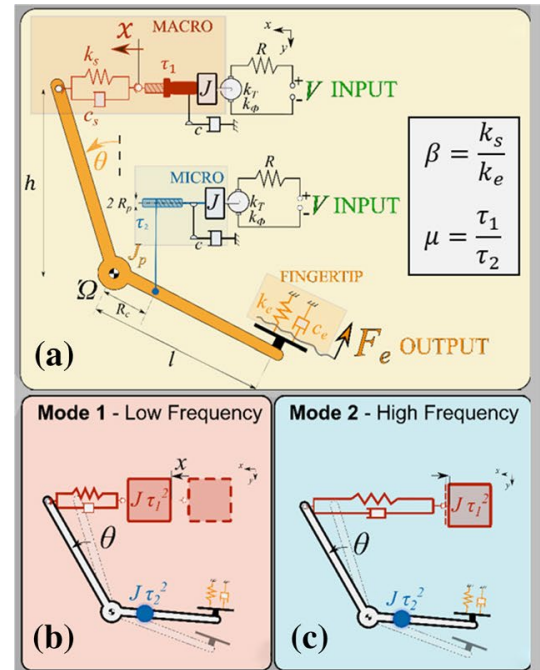

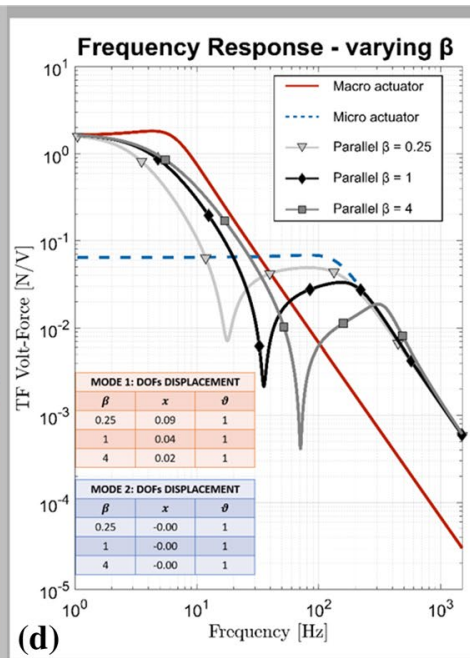

(d)

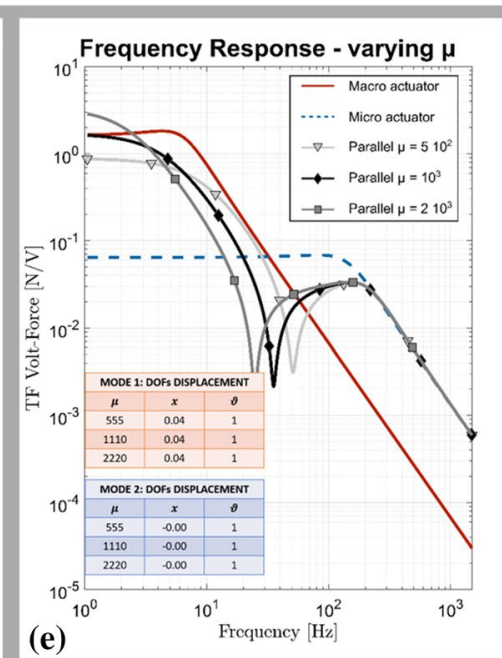

(e) parameter on the magnitude of the frequency response function of the actuation; e effect of the $\mu$ parameter on the magnitude of the frequency response function of the actuation. A small damping factor was introduced for the plots (d) and (e) to improve readability 
to the pinned link through a direct wire transmission. One end of the wire is wrapped on the motor output pulley with a radius $R_{p}$, while the opposite end is fixed to a capstan of the moving link at a distance $R_{c}$ from $\Omega$. At a first approximation, the elasticity of the wire is neglected and dynamics of the actuator is governed by a transmission ratio $\tau_{2}$, equal to the ratio $R_{p} / R_{c}$. Being $\tau_{1}>>\tau_{2}$ the notation macro-micro is adopted to distinguish the actuators.

In the design process, for a given fingertip stiffness $k_{e}$, the parallel elastic stiffness is expressed in the form of the adimensional coefficient $\beta$ :

$\beta=\frac{k_{s}}{k_{e}}$

If we suppose that encumbrance limits determine the value of $\tau_{2}$, the choice of the first transmission ratio $\tau_{1}$ can be normalized by the parameter $\mu$ :

$\mu=\frac{J \tau_{1}^{2}}{J_{p}+J \tau_{2}^{2}}$

Typically, the inertia $J_{p}$ results much lower than the perceived inertia of the motor, hence $\mu \sim \tau_{1}^{2} / \tau_{2}^{2}$.

Lastly, a parameter $\lambda$ is defined to synthesize the geometry of the device:

$\lambda=\frac{h}{l}$

In the following paragraph, the performance of the parallel elastic actuation will be discussed with respect to $\beta$ and $\mu$, while $\lambda$ will be kept fixed. Value of the parameters used in the developed model is shown in Table 1.

\subsection{Theoretical model}

An analytical model of the system was developed to obtain a basic understanding of the interaction between the two actuators and the finger, conceptualizing the role of the two main design parameters of the system: the reduction ratio and the stiffness of the coupling element. To this aim, damping and non-linear effects were not included in the analytical model, and were considered only in the following numerical model.

Design principle is derived through the analysis of the input-output transfer function. Namely the input
Table 1 Parameters used in the model of the system

\begin{tabular}{lll}
\hline Parameters & Value & Unit \\
\hline System parameter & & \\
$k_{e}$ & 2000 & $\mathrm{~N} / \mathrm{m}$ \\
$c_{e}$ & 20 & $\mathrm{Ns} / \mathrm{m}$ \\
$\beta$ & 1 & $/$ \\
$\mu$ & 1000 & $/$ \\
$J$ & $5 \times 10^{-7}$ & $\mathrm{~kg} \times \mathrm{m}^{2}$ \\
$\tau_{1}$ & 12566 & $/$ \\
$\tau_{2}$ & 10 & $/$ \\
Motor parameters & & \\
$R$ & 15.4 & $\Omega$ \\
$K_{t}$ & 0.002 & $\mathrm{Nm} / \mathrm{A}$ \\
$J_{m}$ & $7 \times 10^{-9}$ & $\mathrm{~kg} \times \mathrm{m}^{2}$ \\
\hline
\end{tabular}

are the driving voltages $V$ of the DC servo motors and the output is the force $F_{e}$ exerted on the fingertip.

In Fig. 2 are reported the TF of the separate macromicro actuators for comparison with the parallel architecture. Each one of these single-input singleoutput transfer function is characterized by the static gain and the bandwidth. It is immediate to verify that the static gain of the macro motor is in the ratio $\tau_{1} / \tau_{2}$ with respect to the micro, while the bandwidths are in the ratio $\sqrt{\left(J_{p}+J \tau_{2}^{2}\right) /\left(J_{p}+J \tau_{1}^{2}\right)} \sim \tau_{2} / \tau_{1}$. As a consequence, the macro actuator features low bandwidth and high output force $(1 \sim 5 N)$. Conversely, the micro actuator achieves high-bandwidth $\left(\sim 10^{2} \mathrm{~Hz}\right)$ but provides low maximum amplitude interaction.

For the parallel actuation, a 2 input- 1 output TF is adopted which can be expressed by the following Eq. 4

$H(\omega)=\frac{k_{T}}{R} \frac{\tau_{2}}{\mu^{2}} \frac{l k_{e}\left[\beta k_{e}(\lambda \mu l+1)-\mu^{2} J \tau_{2}^{2} \omega^{2}\right]}{J^{2} \tau_{2}^{4}\left(\omega_{1}^{2}-\omega^{2}\right)\left(\omega_{2}^{2}-\omega^{2}\right)}$

where $\omega_{1}$ and $\omega_{2}$ are the natural frequencies of the 2-dof system poles.

In particular, when $\mu>10$ it results that the high frequency pole may be estimated by the following approximated expression:

$\omega_{2}=\sqrt{\frac{\left(\beta \lambda^{2}+1\right) l^{2} k_{e}}{J \tau_{2}^{2}}}$ 
In the associated mode, Fig. 2c, the pinned plate oscillates between the elastic element and the fingertip, while the macro-motor is practically still.

The mode associated to the low-frequency pole involves both dofs, as pictured in Fig. 2b. Besides, when also $\beta>>1$ a simple mathematical expression is found for the low-frequency pole:

$\omega_{1}=\sqrt{\frac{\beta k_{e}}{\mu^{2} J \tau_{2}^{2}}}$

meaning that the macro-motor bounces on the elastic element.

The static gain of this TF is $H_{0}$ :

$H(\omega=0)=\frac{k_{T}}{R} \lambda \tau_{2} \mu$

and it coincides with the static gain of the TF of the macro actuator. This TF is also characterized by the presence of a zero (anti-pole) whose frequency $\omega_{Z}$ can be estimated by:

$\omega_{Z}=\sqrt{\frac{\beta k_{e}(\lambda \mu l+1)}{\mu^{2} J \tau_{2}^{2}}}$

Figure 2 reports a parametric study of the TF $H$ by varying $\beta$ and $\mu$.

Figure $2 \mathrm{~d}$ reports the effect of varying $\beta$ in a range of possible values while keeping $k_{e}$ and all the other parameters fixed. By increasing $\beta$ (that is by increasing the stiffness of the elastic element), the TF to the TF of the micro actuator. The $\omega_{Z}$ increases.

Figure 2e reports the effect of varying $\mu$ in a range of possible values while keeping $J \tau_{2}^{2}$ and all the other parameters fixed. By increasing $\mu$, the static gain increases, while the $\omega_{Z}$ decreases. What is more, the high-frequency behavior seems to be less sensitive, being the TF very close after the $\omega_{Z}$ and near to the profile of the micro-actuator TF. Although not considered in the theoretical model, a small damping factor was introduced for the plots d) and e) to improve readability.

Equations of the analytical model presented above did not take into account damping and other non-linear factors such as friction and backlash. A numerical model was then developed to better simulate the additional complexity of the real device. Details of the simulated model are described in Sect. 3.1.

\section{Design of the fingertip haptic device}

The parallel elastic actuation approach has been conceived for implementation in a miniaturized fingertip device as shown in the general scheme of Fig. 1. Two actuators of the same size are implemented, varying only the mechanical reduction between each of them and the moving plate. Actuators were two Minebea K30 micro DC motors, diameter $8 \mathrm{~mm}, 5 \mathrm{~V}$ nominal voltage.

A lead-screw mechanism connects the output of the first actuator to the elastic coupling element and in turn to the moving link. The lead-screw mechanism has been chosen due to its high mechanical reduction without and low noise if compared to gear trains. The screw transmission was fabricated in Teflon (PTFE) for the nut, and Polyoxymethylene (POM) for the screw, with an M3 thread.

The second actuator is attached to the moving link through of a wire transmission: a capstan (radius $5 \mathrm{~mm}$ at the moving link is connected by a tendon to a pulley with low radius (radius $1 \mathrm{~mm}$ ) at the output shaft of the motor. It constitutes a low-reduction ratio, reversible mechanical transmission. A silicone tube was used to fabricate the coupling elastic element: after preliminary experiments the soft material was preferred with respect to a steel spring due to the introduction of damping. A contactless reflectance position sensor was been embedded between the moving plate and the body, measuring displacement of the moving link with the contact plate (measured noise of $0.01 \mathrm{~mm}$ in the middle point of the measuring range). It allows to better control the reduced actuator in position, overcoming the non-backdrivability of the screw reduction.

Overview of the design and arrangement of the mechanical parts is shown in Fig. 3. Device design has been studied in order to minimize lateral interference with other fingers and encumbrance within the hand workspace. The final device is $16 \mathrm{~mm}$ wide, with $21 \mathrm{~g}$ total mass.

\subsection{Device simulation}

A simulation of the system was developed in order to better evaluate more complex and non-ideal behaviors of the implemented device, introduced i.e. by friction of the screw reduction and compliance of the physical tendon transmission. The simulation was developed 
Fig. 3 Mechanical components of the Parallel Elastic Haptic Thimble

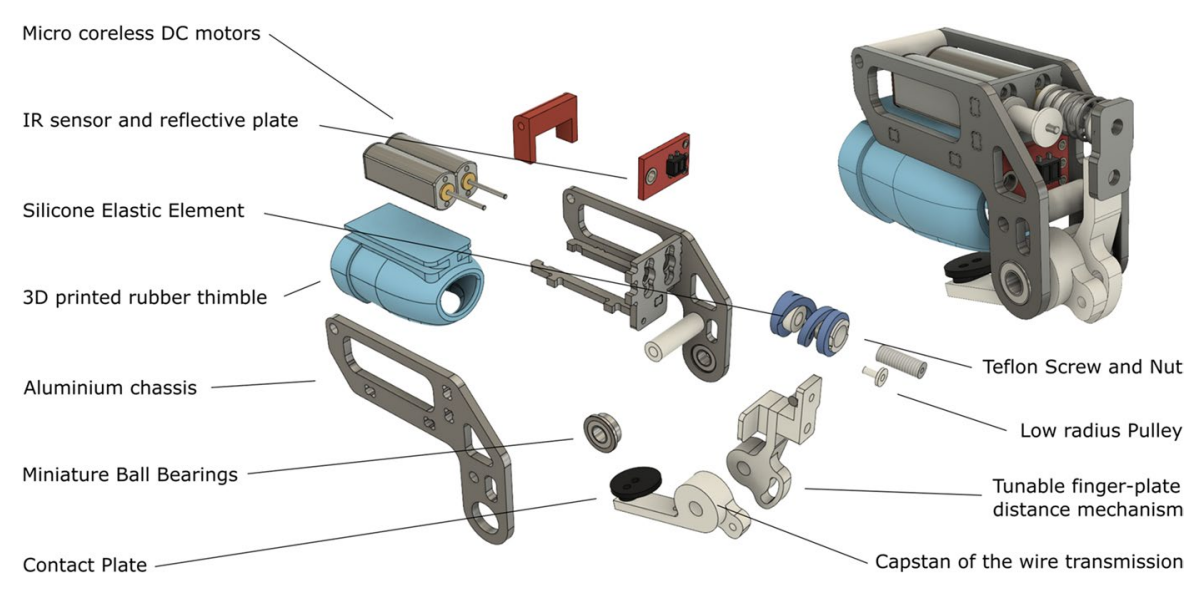

using Matlab Simulink. The model included the following features of the physical system: electrical and mechanical simulation of the DC micromotors (electrical and mechanical time constants and back-electromotive force BEMF), static and dynamic friction of the screw reducer, stiffness of the elastic coupling and stiffness of the transmission of the direct actuator. In order to properly simulate these aspects, experimental characterization of each part of the system was performed (details are described in Sect. 4.2). We explored simulated behavior of the device at varying of two design parameters in particular: the stiffness of the elastic coupling and the gear reduction of the two motors.

\section{Experiments and results}

In this section we present results obtained from the implemented prototype (Fig. 4) and from the simulated model (Figs. 5 and 6).

\subsection{Simulation results}

Results of the numerical model regarding frequency response of the system at varying stiffness of the elastic coupling are shown in Fig. 6. The black solid line represents the frequency response with both motors active (same input signal) and with parameters corresponding to the real device implementation. Profile of the frequency response resembles the theoretical one shown in Fig. 2, with smoother peaks due to presence of damping (please note that a small damping factor was used in Fig. 2 for readability of the plot, although

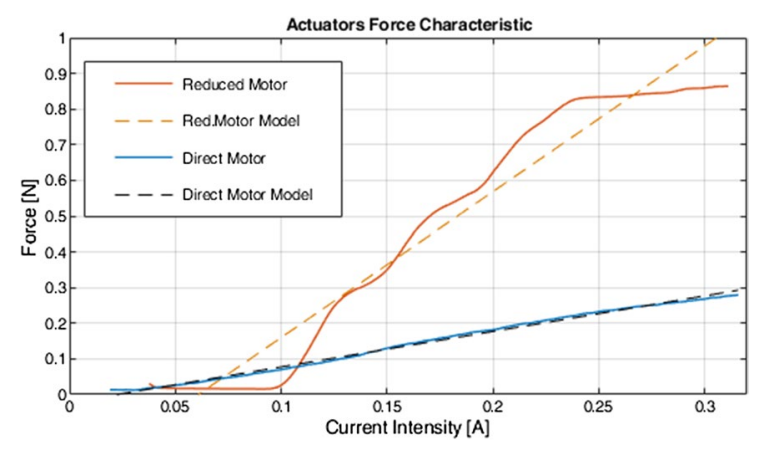

Fig. 4 Force characterization of the two actuators, evidencing the different static force output at the fingerpad plate

damping was not considered in the theoretical model). Regarding variation of the elastic coupling, results are shown activating each actuator at a time. Figure 6 shows that a more stiff coupling improves response of the reduced motor in the static-to low frequency range. Conversely, a more compliant coupling enhances the overall frequency response of the direct motor, which is less rigidly coupled to the output of the slow reduced motor. However, graph shows that at frequency higher than the cutoff frequency, response of the direct motor is not affected by variations of the coupling stiffness: this happens due to the prevalent effect of the non-rigid transmission of the direct motor. Regarding variations of the reduction ratio, Fig. 5 shows the frequency response with both actuators active in black solid line (same response as in Fig. 6, then response with only one actuator active at time is shown by varying reduction ratio of the active actuator only. Results show that an increase in 


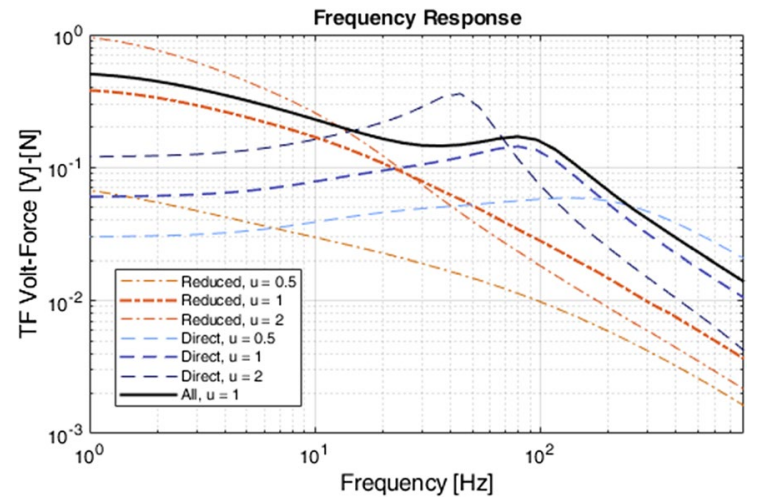

Fig. 5 Simulated results varying the reduction ratio of the two motors

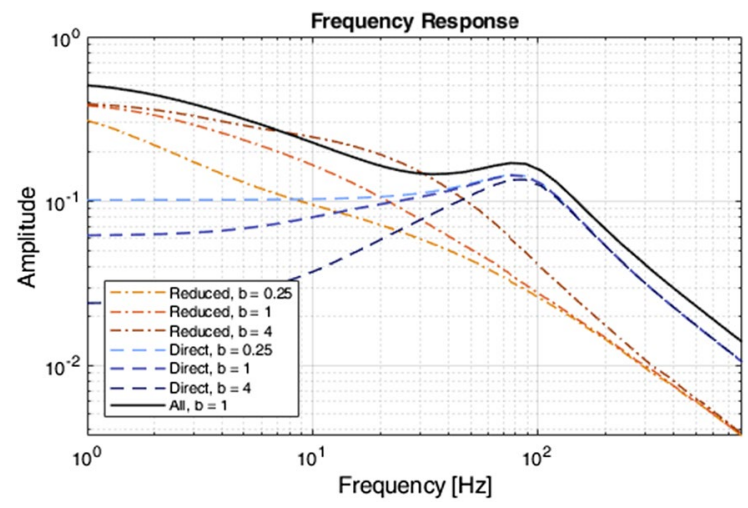

Fig. 6 Simulated results varying the stiffness of the elastic element

the reduction ratio, as expected enhances the static to low-frequency response for both actuators. On the other side, it limits the output bandwidth decreasing the cut-off frequency. Also, a higher reduction ratio of the direct motor increases the resonant peak, resulting in a non-flat response in the working bandwidth.

\subsection{Experimental results}

The experimental activity was conducted to characterize frequency response, output forces and haptic rendering performance of the implemented prorotype. A compact force sensor (Optoforce 10N with resolution of $1 \mathrm{mN}$ ) was mounted in place of the rubber thimble (Fig. 7 left) using a custom holder. A microcontroller board (Teensy 3.6) executed the low level control of the device, to acquire the reflectance position sensor,

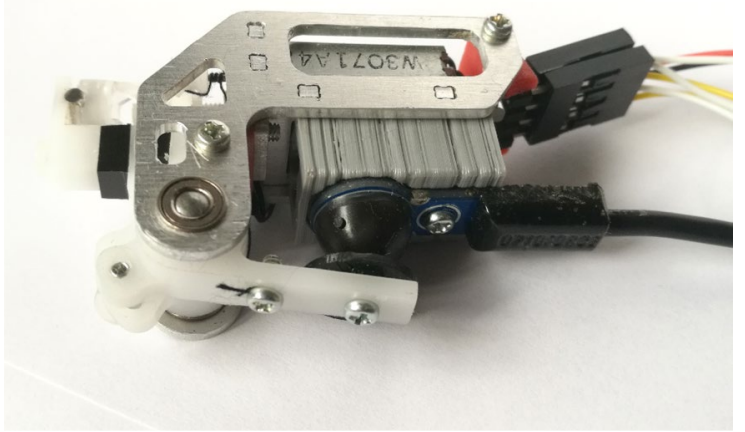

Fig. 7 Experimental setup with the force sensor mounted in place of the fingertip for characterization of the device

and to drive motors by a dual H-bridge IC (Texas Instruments DRV8835). Sample time of the low-level control loop was $4 \mathrm{KHz}$.

The current intensity-force characteristics of the two actuators was experimentally measured and shown in Fig. 4. The first motor shows a higher output force, presenting non-linearity due to friction of the lead-screw mechanism. The second actuator shows a lower output force and a linear characteristic.

The characterization curve of the reduced motor was also used to estimate the static friction and the dynamic friction coefficient of the screw transmission. Finally, stiffness of the whole direct motor transmission (including the motor output shaft, the cable and the plate link) was experimentally measured by dividing the force measured at the plate by an externally-imposed angular displacement at the motor. Linearized results provided an equivalent lumped stiffness of the direct transmission of $9.72 \mathrm{~N} / \mathrm{mm}$.

Frequency response of the device was measured by providing a feed-forward voltage reference chirp signal, logarithmically increasing from 1 to $800 \mathrm{~Hz}$. A reference constant offset was set in order to keep the plate always in contact with the sensor. Stiffness of the optical force sensor was experimentally measured and equal to $30.1 \mathrm{~N} / \mathrm{mm}$.

The experimentally measured frequency response is shown in Fig. 8. The black solid line represent the condition with both motors activated (same input signal) while the blue and orange lines represent the frequency response obtained with only the direct or the reduced motor active respectively. Profile resembles 


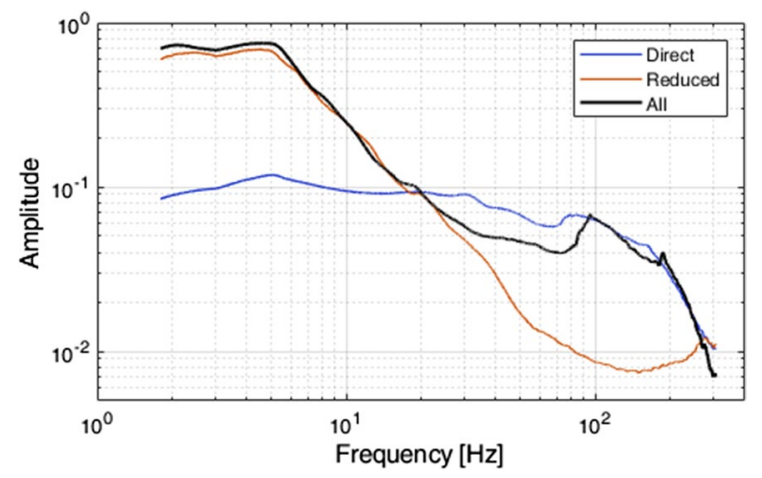

Fig. 8 Experimental frequency response of the device using a feed-forward chirp input at the direct, reduced and both motors respectively

simulation and theoretical results, although it has to be considered the stiffness of the force sensor was significantly different from that of the simulated finger. Similarly to the simulation, a peak is present at $96 \mathrm{~Hz}$. A second one appears at $188 \mathrm{~Hz}$, probably due to the resonant frequency of the moving plate lever.

A second experimental activity was then conducted in order to explore quality of the haptic rendering with the device worn at a finger (in place of the force sensor). The embedded position sensor was used to tune a position control loop for the reduced actuator. The closed loop was needed to improve stability of the screw-reduced actuator with respect to the pure feed-forward control used in the frequency response estimation.

The device was then worn at the experimenter's index finger. A texture pattern ("Sandpaper 100") was selected from the online available texture library of the Penn Haptic Texture Toolkit [4], and used as reference input signal. In order to adapt the texture pattern to the range of the device, reference signal for the direct actuator was high-pass filtered at the first cutoff frequency of the frequency response of the device. With this approach, the direct actuator was not in charge of the static components of the normal force, which in the reference signal had a noticeably high intensity. Two experimental conditions were performed: with both actuators enabled, and with the reduced actuator only. Since the user's finger was in place of the external force sensor, measured data refers to the embedded position sensor. Results are shown in Fig. 9. Frequency response shown in Fig. 9 (right), highlights the effect of both actuators enabled, obtaining a response closer to the reference. Details of Fig. 9 (middle) shows a better visualization of the higher frequency components in the time domain. The introduction of the direct drive actuator coupled in parallel, obtains more crisp and dynamic output
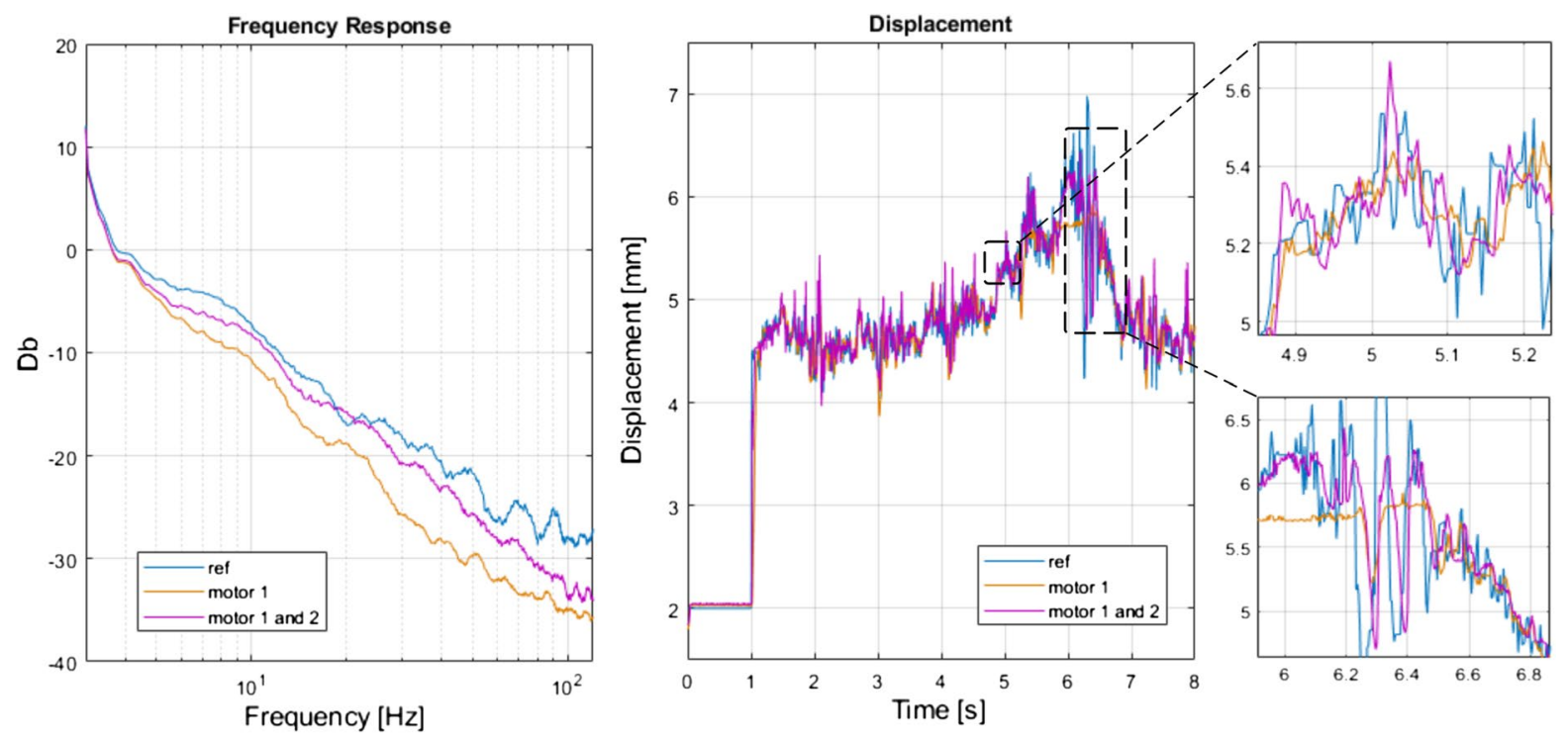

Fig. 9 Bench test of the device rendering a sample texture (sandpaper) with contact transition and non-zero constant component of the normal force 
signals, whereas the reduced actuator only, in particular at the higher indentation levels (top detail), shows a more flat response.

\section{Discussions}

The developed theoretical model implemented three state variables and was able to depict the overall behavior of the device: two cutoff peaks are clearly visible from the frequency response: the first cutoff at about $10 \mathrm{~Hz}$ highlights components of the reduced actuator transmitted to the output plate. The second cutoff frequency indicate the bandwidth limit of the fast (direct) actuator: its frequency placement is related to the residual compliance of the tendon transmission. Amplitude of the static gain of the device and at the second peak in the frequency response depends on the stiffness of the coupling between the two parallel actuators. It emerges that increasing the stiffness of the coupling element over the fingerpad stiffness $(\beta>1)$, does not enhance response of the device: range of the lower frequency components are not significantly extended, and amplitude of the higher frequency components is noticeably reduced (Fig. 2). Varying the reduction ratio can instead modify the static and low frequency gain of the device, although the interaction between the two poles in the frequency response becomes not clearly visible in the theoretical model due to the presence of the zeros.

More complex behaviors (compliance and friction of the non-ideal transmissions) have been evaluated through the simulation of the numerical model. With respect to the theoretical model, response was different especially in the frequency range between the two poles of the system, due to the simulated compliance of the transmission cable and to the higher energy losses introduced by friction. Simulation of different stiffness for the coupling element showed a trade-off between signal components transmitted by the two motors: high stiffness of the coupling element enhances force transmission of the reduced motor but limits the contribution of the direct motor. However, in the simulated results the higher frequency components seemed less affected: this can be explained by the high static friction introduced at the screw transmission, making the reduced motor practically still in any condition when the direct motor only is active.
Increasing the reduction ratio for the reduced motor, results into an increase of the static force with a lower cutoff frequency. Increasing the reduction ratio of the "direct" motor instead, produces a shift of the second cutoff frequency with an increase of the peak amplitude and a less flat response for the higher frequency components.

Force characterization and frequency response of the final prototype showed the desired different behavior of the two actuators (same motors with different reduction) in complementary frequency ranges. The sample texture rendering experiment showed also the output rendering was closer to the reference when both actuators were enabled. A drawback of the final implementation was the relatively limited cutoff frequency of the direct motor $( \pm 100 \mathrm{~Hz})$ due to the compliance of the miniaturized tendon transmission and to the mechanical time constant of the micro-sized gearmotor. In fact, the transmission implemented one branch only, and no pre-tensioning of the tendon was possible. On the other hand, a two-branches transmission with pre-tensioning would not be suitable for a micro-motor, since it mounts only bushes (no ball bearings) at the output shaft.

\section{Conclusions}

The novel design of a wearable fingertip device implementing a parallel elastic mechanism was proposed. The method has been proposed to better comply with requirements of wearable devices, in terms of limited encumbrance and mass, and features of the haptic rendering, involving static to high frequency force components. In this work we investigated whether a parallel actuator design can better match frequency response to the typical haptic rendering requirements. A first motor, with higher mechanical reduction, provides static to low frequency components of the output force, (i.e. grasping modulation or normal forces in surface exploration). The second actuator, with lower reduction, enhances rendering of high frequency tactile cues, which typically have a reduced amplitude with respect to the static and slow force components. A coupling elastic element is needed in order to allow both actuators to operate.

Through the analytical and simulated model, we explored the resulting interaction between the two actuators at different values of certain design 
parameters, i.e. the coupling stiffness and the different reduction ratios. Results showed how the desired response of the device can be modulated by the above design parameters, obtaining different balance of the slow and high frequency components in the rendered signal.

The final experimental activity evaluated how the presented method can be effectively implemented in a miniaturized fingertip haptic device, featuring a balance between bandwidth and maximum output force. The proposed method can result in more compact wearable devices with improved energy efficiency and capabilities, in terms of quality of the output signal and hi-fidelity rendering.

Funding This work was funded by the Project "TELOS - Tailored neurorehabilitation thErapy via multi-domain data anaLytics and adapative seriOus games for children with cerebral palSy", which is funded under the call "Bando Ricerca Salute 2018” of Tuscany Region, Italy, (CUP J52F20001040002).

\section{Declaration}

Conflict of interest The authors declare that they have no conflict of interest.

Open Access This article is licensed under a Creative Commons Attribution 4.0 International License, which permits use, sharing, adaptation, distribution and reproduction in any medium or format, as long as you give appropriate credit to the original author(s) and the source, provide a link to the Creative Commons licence, and indicate if changes were made. The images or other third party material in this article are included in the article's Creative Commons licence, unless indicated otherwise in a credit line to the material. If material is not included in the article's Creative Commons licence and your intended use is not permitted by statutory regulation or exceeds the permitted use, you will need to obtain permission directly from the copyright holder. To view a copy of this licence, visit http://creativecommons.org/licenses/by/4.0/.

\section{References}

1. Bensmaia Sliman J, Mark Hollins (2000) Complex tactile waveform discrimination. J Acoust Soc Am 108(3):1236-1245

2. Caldwell DG, Tsagarakis N, Wardle A(1997) Mechano thermo and proprioceptor feedback for integrated haptic feedback. In: Robotics and automation, 1997. Proceedings., 1997 IEEE international conference on, IEEE, vol 3, pp 2491-2496

3. Chinello F, Malvezzi M, Pacchierotti C, Prattichizzo D (2015) Design and development of a 3rrs wearable fingertip cutaneous device. In: Advanced intelligent mechatronics (AIM), 2015 IEEE international conference on, IEEE, pp 293-298

4. Culbertson H, Delgado JJL, Kuchenbecker KJ (2014) One hundred data-driven haptic texture models and opensource methods for rendering on 3d objects. In: 2014 IEEE haptics symposium (HAPTICS), IEEE, pp 319-325

5. Fani S, Ciotti S, Battaglia E, Moscatelli A, Bianchi M (2017) W-fyd: a wearable fabric-based display for haptic multi-cue delivery and tactile augmented reality. IEEE Trans Haptics 11(2):304-316

6. Gabardi M, Chiaradia D, Leonardis D, Solazzi M, Frisoli A (2018) A high performance thermal control for simulation of different materials in a fingertip haptic device. In: International conference on human haptic sensing and touch enabled computer applications, Springer, pp 313-325

7. Gabardi M, Solazzi M, Leonardis D, Frisoli A (2016) A new wearable fingertip haptic interface for the rendering of virtual shapes and surface features. In: 2016 IEEE haptics symposium (HAPTICS), IEEE, pp 140-146

8. Gallo S, Rognini G, Santos-Carreras L, Vouga T, Blanke O, Bleuler H (2015) Encoded and crossmodal thermal stimulation through a fingertip-sized haptic display. Front Roboti AI 2:25

9. Jenmalm P, Dahlstedt S, Johansson RS (2000) Visual and tactile information about object-curvature control fingertip forces and grasp kinematics in human dexterous manipulation. J Neurophysiol 84(6):2984-2997

10. Kuchenbecker KJ, Gewirtz J, McMahan W, Standish D, Martin P, Bohren J, Mendoza PJ, Lee DI (2010) Verrotouch: high-frequency acceleration feedback for telerobotic surgery. In: International conference on human haptic sensing and touch enabled computer applications, Springer, pp 189-196

11. Leonardis D, Gabardi M, Solazzi M, Frisoli A (2020) A parallel elastic haptic thimble for wide bandwidth cutaneous feedback. In: International conference on human haptic sensing and touch enabled computer applications, Springer, pp 389-397

12. Leonardis D, Solazzi M, Bortone I, Frisoli A (2015) A wearable fingertip haptic device with 3 dof asymmetric 3-rsr kinematics. In: 2015 IEEE world haptics conference (WHC), IEEE, pp 388-393

13. Leonardis D, Solazzi M, Bortone I, Frisoli A (2016) A 3-rsr haptic wearable device for rendering fingertip contact forces. IEEE Trans Hptics 10(3):305-316

14. Lu T, Pacoret C, Hériban D, Mohand-Ousaid A, Regnier S, Hayward V (2016) Kilohertz bandwidth, dual-stage haptic device lets you touch brownian motion. IEEE Trans Hptics 10(3):382-390

15. Morrell JB, Kenneth SJ (1998) Parallel-coupled micromacro actuators. Int J Robot Res 17(7):773-791

16. Pacchierotti C, Prattichizzo D, Kuchenbecker KJ (2015) Cutaneous feedback of fingertip deformation and vibration for palpation in robotic surgery. IEEE Trans Biomed Eng 63(2):278-287

17. Pacchierotti C, Sinclair S, Solazzi M, Frisoli A, Hayward V, Prattichizzo D (2017) Wearable haptic systems for the fingertip and the hand: taxonomy, review, and perspectives. IEEE Trans Haptics 10(4):580-600 
18. Shin D, Sardellitti I, Khatib O (2008) A hybrid actuation approach for human-friendly robot design. In: 2008 IEEE international conference on robotics and automation, IEEE, pp 1747-1752

19. Solazzi M, Frisoli A, Bergamasco M (2010) Design of a novel finger haptic interface for contact and orientation display. In: Haptics symposium, 2010 IEEE, IEEE, pp 129-132

20. Wall SA, Harwin W (2001) A high bandwidth interface for haptic human computer interaction. Mechatronics 11(4):371-387

21. Wang D, Ohnishi K, Xu W (2019) Multimodal haptic display for virtual reality: a survey. IEEE Trans Ind Electron 67(1):610-623

22. Wiertlewski M, Lozada J, Hayward V (2011) The spatial spectrum of tangential skin displacement can encode tactual texture. IEEE Trans Robot 27(3):461-472
23. Yau JM, Olenczak JB, Dammann JF, Bensmaia SJ (2009) Temporal frequency channels are linked across audition and touch. Curr Biol 19(7):561-566

24. Zinn M, Khatib O, Roth B, Salisbury JK (2008) Large workspace haptic devices-a new actuation approach. In: 2008 Symposium on haptic interfaces for virtual environment and teleoperator systems, IEEE, pp 185-192

Publisher's Note Springer Nature remains neutral with regard to jurisdictional claims in published maps and institutional affiliations. 